\title{
Virtualizing Power Cords by Wireless Power Transmission and Energy Harvesting
}

\author{
Yoshihiro Kawahara ${ }^{1}$, Wei Wei ${ }^{1}$, Yoshiaki Narusue ${ }^{1}$, Ryo Shigeta ${ }^{1}$, \\ Tohru Asami ${ }^{1}$, and Manos Tentzeris ${ }^{2}$ \\ 1 The University of Tokyo, 7-3-1 Hongo, Bunkyo-ku, Tokyo 113-8656, Japan \\ 2 Georgia Institute of Technology, $855^{\text {th }}$ street NW, Atlanta, GA 30308, USA
}

\begin{abstract}
In this paper, we introduce two different approaches for the virtualization of power cords for electrical devices. The first approach is a new concept for routing electric power by wireless transfer on two-dimensional surfaces, such as floors and walls. Unlike any other existing wireless power transfer scheme, this method can deliver electric power over a wide range with minimal loss. We realize this method using multi-hop displacement of a magnetic antenna array. Each array element can be selectively resonated with adjacent elements to deliver power without physical contact. The second approach utilizes farfield RF energy harvesting. Using an efficient voltage multiplier and adaptive software-based control, it is possible to operate low-power wireless sensors continuously.
\end{abstract}

Index Terms - Wireless power transmission, RF energy harvesting.

\section{INTRODUCTION}

Wireless communication has permeated our daily lives. Most portable electronic devices such as laptop computers and mobile telephones can communicate wirelessly. However, users are still forced to plug in a power cord to charge an electrical device. This significantly inhibits mobility and usability in a ubiquitous computing environment. Even though an energy storage technology including batteries and capacitors advances, the problem of a power supply remains an important issue. The cost of replacing or recharging the batteries of wireless sensor nodes is extremely high when a number of sensor nodes are deployed in the field. Hence, there is a general need to remove the electrical cords from rooms at low cost.

If the wireless communication technology can be called the virtualization of the communication line, wireless power transmission is the virtualization of power cords. Our research group has been working on the virtualization of power cords using two different methods. The first approach routes electric power by wireless transfer on two-dimensional surfaces such as floors and walls by using magnetic coupling resonators. The second approach utilizes far-field RF energy harvesting that exploits ambient RF signals as a power supply for low-power electronics devices. This paper is organized as follows. First, we introduce a virtual power routing concept in
Section II. Then, we introduce the RF energy harvesting approach and its enabling technologies in Section III.

\section{2D WIRELESS TRANSMISSION BY MAGNETIC RESONANT COUPLING}

We propose a two-dimensional wireless power transfer (WPT) system using magnetic resonant coupling [1]. In this system, we place multiple resonators in a tiled pattern on the floor or the wall. By switching the impedance of each resonator element, the system can route power throughout an entire room to designated devices only and with a certain resonant efficiency. By establishing a virtual power line to a device, it is possible to suppress unnecessary feeding to unused devices. For ease of deployment, the resonator element is sized to fit in with typical modular carpet tiles. Because each element is magnetically coupled to the adjacent elements, there is no need to physically connect them with wires or conductive contacts. This property, different from that of any other two-dimensional power transfer system, significantly decreases the deployment cost and increases the layout flexibility. Moreover, the resonators can resonate with each other in both the vertical and the horizontal directions. This property makes it possible to extend the power supply range to each spatial surface. For instance, it is possible to create a virtual path between the floor and a tabletop without a physical conduit.

Several technical challenges must be solved to realize this concept. The first challenge is the design of the optimal configuration of the resonators. In existing WPT systems, resonators are aligned on top of each other. In our system, as in Fig. 1, the resonators must be tiled adjacently, which results in weaker coupling between resonators. In terms of minimizing the thickness, the single resonator shown on the left-hand side of Fig. 2 is useful. However, we have shown that our proposed double-sided spiral resonator can extend the effective transmission range by allowing an increase in the thickness of $5 \mathrm{~mm}$ (Double Spiral-5) to 10 mm (Double Spiral-10), as in Fig. 3. Because two pieces of spiral coils with the same size were utilized to form a double-spiral resonator, the magnetic flux in the coils is 
almost doubled compared with a single spiral resonator. The coupling coefficients at a distance of $5 \mathrm{~cm}$ apart were $0.12,0.19$, and 0.23 , respectively. The increase in the coupling coefficient resulted in an increase in the transmission range.

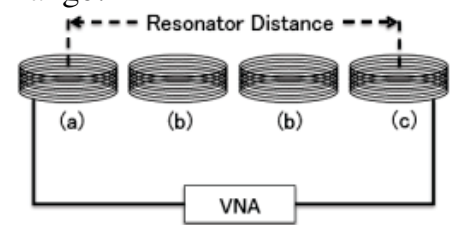

Fig. 1. Relay resonators.

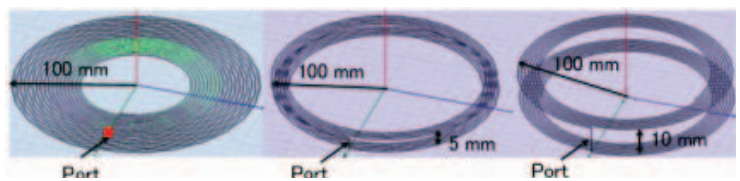

Fig. 2. Configuration of the multi-hop resonators.

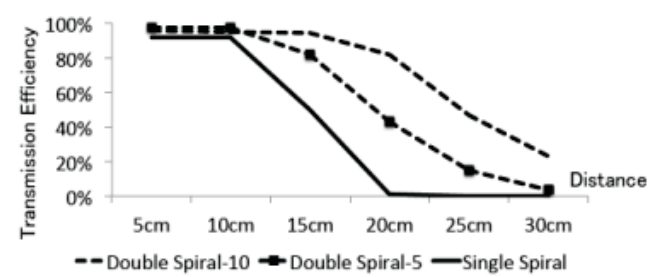

Fig. 3. Measurement result of maximum transmission efficiency $\left(\left|\mathrm{S}_{21}\right|^{2}\right)$ of the resonators.

The second challenge of this approach is impedance matching. When $N$ resonators are "strongly coupled," the resonant frequency is split into $N$ modes, depending on the coupling coefficient. In order to acquire maximum efficiency at a certain fixed frequency, for instance, the resonant frequency of the resonators, the BPF theory was utilized to improve the deployment of the two-hop system, which consists of a feeding, relay, and receiving resonators [2]. We extended the BPF theory to a multi-hop deployment system (more than two hops). By adjusting the distance between resonators, it is possible to suppress the split and realize flat frequency characteristics around the self-resonant frequency of the resonators. The details of the impedance-matching conditions are introduced in [1]. We also improved this method so that the impedance is always matched regardless of the number of hops, at the expense of reduced bandwidth. The details of this approach are introduced in [6].

\section{POWER HARVESTING FROM AMBIENT RF SigNALS}

Wireless power transmission using magnetic resonance is suitable for indoor devices such as electrical appliances. However, this method is not suitable for covering a large area in an outdoor environment. RF signals available in the air are an alternative energy source because radio waves are ubiquitous in our daily lives. However, the difficulty of this approach is that the power density of the signal is extremely low for the operation of electrical devices. For instance, communication terminals such as mobile phones can communicate at a few dozen femtowatts $(-110 \mathrm{dBm})$. Even if it becomes possible to harvest electrical energy from the source, the electrical energy of a few dozen femtowatts is not useful for most electrical devices. However, for low-power applications such as wireless sensor networks, there are some signal sources that can be utilized to harvest energy.

For example, the transmitted power from a TV broadcast station is on the order of several kilowatts, but the received power ranges from microwatts to milliwatts depending on the distance from the tower. Our measurement results for the past three years indicate that the average amount of RF energy is a few microwatts at most places in downtown Tokyo, several kilometers away from a TV tower [3]. In certain places, the RF energy reaches at most several hundred microwatts. A sensor node generally consumes more than $10 \mathrm{~mW}$ of power while transmitting a packet over a wireless link. However, the operation of wireless sensor networks is often intermittent, meaning that if the energy harvested is stored in a battery or a capacitor, a node would be able to operate using the stored energy.

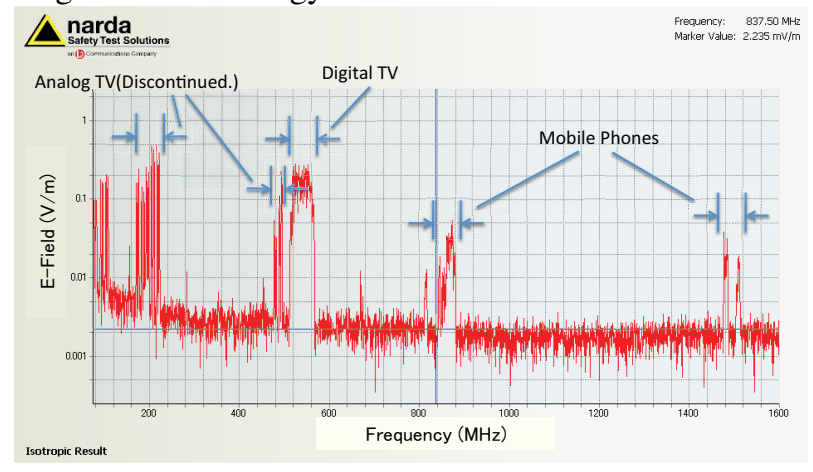

Fig. 4. Spectrum in downtown Tokyo, $6.5 \mathrm{~km}$ from TV Tower.

Fig. 4 shows the spectrum measurement results in downtown Tokyo (POI) using a NARDA SRM-3000 radiation meter and indicates significant wireless activity in the UHF band between 480 and $580 \mathrm{MHz}$ attributed to digital TV broadcasts. Currently, analog TV broadcasts in the VHF band have been terminated. Mobile phone signals are not useful because the power levels significantly vary depending on the time of day.

The antenna shown in Fig. 5 is a folded dipole structure resonating at $515 \mathrm{MHz}$, the frequency at which digital TV service is provided. The antenna length was optimized to be about half of a wavelength long to maximize its gain and effective aperture area, which would increase the 
voltage at its terminals and the incident wireless power collected. The antenna width was optimized to achieve a wideband impedance of close to $50 \Omega$ in order to minimize reflection losses with a matched RF-to-DC charge pump circuit. The measured $\left|S_{11}\right|$ of the antenna shows a reflection loss of lower than $-15 \mathrm{~dB}$ from 467 $\mathrm{MHz}$ to well beyond $580 \mathrm{MHz}$, ensuring that $97 \%$ of the transduced wireless TV signal power can reach the matched RF-to-DC converter circuit.

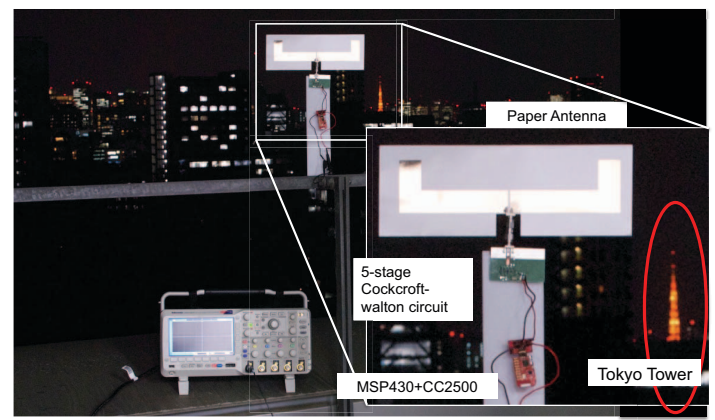

Fig. 5. The rectifier and antenna.

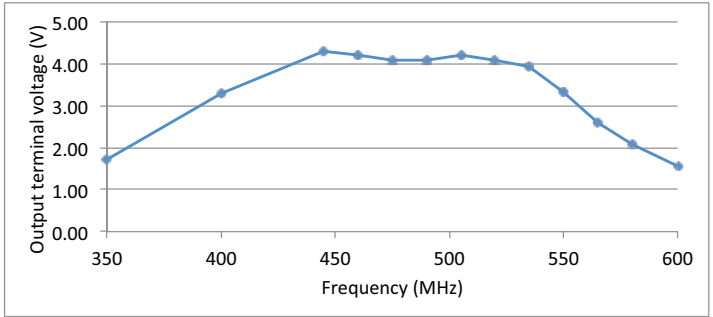

Fig. 6. Output terminal voltage versus frequency.

The incoming power transduced across the antenna is in the RF form and induces a very low voltage across the antenna terminals. Being able to harness this power requires rectification and stepping-up to a voltage of $1.8 \mathrm{~V}$ or higher, the voltage at which most electronic devices, such as embedded processors and transceivers, used for wireless sensing operate. A 5-stage RF charge pump circuit was designed and optimized for this task [4]. A $100-\mu \mathrm{F}$ low-leakage capacitor was used as the energy storage charge tank to power the sensor node. Fig. 6 shows the output terminal voltage of the rectifier at different frequencies when the input power is fixed to -3 $\mathrm{dB}$. These results show that the rectifier output is maximized for the entire digital $\mathrm{TV}$ band. In a real environment, the input signal is the result of the integration of nine channels. In addition, the efficiency of rectification changes depending on the load and charge level of the energy storage capacitor. At the balcony of the POI, $6.5 \mathrm{~km}$ away from TV tower, the $100-\mu \mathrm{F}$ capacitor connected to the rectifier is charged to $2.9 \mathrm{~V}$ in $3 \mathrm{~min}$, which means that $0.45 \mathrm{~mJ}$ was collected. To maximize the duty cycle of the sensor node, we implemented an adaptive duty cycle control. This method maximizes the sensing rate taking into account a leakage problem that has never been considered before. We defined the cost minimization problem as an integration of two different costs. One function represents the loss from leakage that linearly increases with the battery level, and the other function expresses the risk to system performance from an energy shortage that is estimated by the stochastic distribution patterns of harvested energy. The LQ tracking method was employed to determine the duty cycle [5].

\section{CONCLUSION}

In this paper, we introduced two different approaches for virtualizing power cords to electrical devices. Because of its nature, wireless power transmission using magnetic resonance is more suitable for indoor applications. In an outdoor environment, ambient RF signals can be exploited for low-power applications such as wireless sensor networks.

\section{ACKNOWLEDGEMENT}

Research projects introduced in this paper have been sponsored by JSPS KAKENHI 2268004 and NEDO.

\section{REFERENCES}

[1] W. Wei, T. Miyasaka, Y. Kawahara, T. Asami, "Maximizing wireless power transmission efficiency with linear deployment resonator array and band pass filter theory," in Adjunct Proc. Pervasive 2012, Newcastle, UK, May 2012.

[2] I. Awai, "Design theory of wireless power transfer system based on magnetically coupled resonators," in Proc. IEEE International Conference on Wireless Information Technology and Systems (ICWITS), pp. 1-4, August 2010.

[3] H. Nishimoto, Y. Kawahara, T. Asami, "Prototype implementation of ambient RF energy harvesting wireless sensor networks," in Proc. IEEE Sensors 2010, pp. 1282$1287,2010$.

[4] R. Vyas, H. Nishimoto, M. Tentzeris, Y. Kawahara, T. Asami, "A battery-less, energy harvesting device for long range scavenging of wireless power from terrestrial TV broadcasts," in Proc. IEEE MTTS International Microwave Symposium (IMS2012), Montreal, Canada, June 2012.

[5] R. Shigeta, Y. Kawahara, R. Vyas, M. Tentzeris, T. Asami, "Ambient RF energy-harvesting sensor node with capacitorleakage aware duty cycle control," in Proc. IEEE Sensors 2012.

[6] Y. Narusue, Y. Kawahara, T. Asami, "Impedance matching method for any-hop straight wireless power transmission using magnetic resonance," RWS2013. 